\title{
QTL Analysis of Resistance to High-Intensity UV-B Irradiation in Soybean (Glycine max [L.] Merr.)
}

\author{
Min Young Yoon ${ }^{1,+}{ }^{,}$Moon Young Kim ${ }^{1,2}$, Jungmin $\mathrm{Ha}^{1,2}$, Taeyoung Lee ${ }^{1}$, Kyung Do Kim ${ }^{3}$ and \\ Suk-Ha Lee ${ }^{1,2, *(1)}$ \\ 1 Department of Plant Science and Research Institute of Agriculture and Life Sciences, \\ Seoul National University, Seoul 08826, Korea \\ 2 Plant Genomics and Breeding Institute, Seoul National University, Seoul 08826, Korea \\ 3 Corporate R\&D, LG Chem, Seoul 07795, Korea \\ * Correspondence: sukhalee@snu.ac.kr; Tel.: +82-2880-4545; Fax: +82-2877-4550 \\ + Present address: Professional Fine Chemical Business Team, Life Science, LG Chem, Seoul 07795, Korea.
}

Received: 4 June 2019; Accepted: 3 July 2019; Published: 4 July 2019

check for updates

\begin{abstract}
High-intensity ultraviolet-B (UV-B) irradiation is a complex abiotic stressor resulting in excessive light exposure, heat, and dehydration, thereby affecting crop yields. In the present study, we identified quantitative trait loci (QTLs) for resistance to high-intensity UV-B irradiation in soybean (Glycine max [L.]). We used a genotyping-by-sequencing approach using an F6 recombinant inbred line (RIL) population derived from a cross between Cheongja 3 (UV-B sensitive) and Buseok (UV-B resistant). We evaluated the degree of leaf damage by high-intensity UV-B radiation in the RIL population and identified four QTLs, UVBR12-1, 6-1, 10-1, and 14-1, for UV-B stress resistance, together explaining $20 \%$ of the observed phenotypic variation. The genomic regions containing UVBR12-1 and UVBR6-1 and their syntenic blocks included other known biotic and abiotic stress-related QTLs. The QTL with the highest logarithm of odds (LOD) score of 3.76 was UVBR12-1 on Chromosome 12, containing two genes encoding spectrin beta chain, brain (SPTBN, Glyma.12g088600) and bZIP transcription factor21/TGACG motif-binding 9 (bZIP TF21/TGA9, Glyma.12g088700). Their amino acid sequences did not differ between the mapping parents, but both genes were significantly upregulated by UV-B stress in Buseok but not in Cheongja 3. Among five genes in UVBR6-1 on Chromosome 6, Glyma.06g319700 (encoding a leucine-rich repeat family protein) had two nonsynonymous single nucleotide polymorphisms differentiating the parental lines. Our findings offer powerful genetic resources for efficient and precise breeding programs aimed at developing resistant soybean cultivars to multiple stresses. Furthermore, functional validation of the candidate genes will improve our understanding of UV-B stress defense mechanisms.
\end{abstract}

Keywords: soybean; UV-B stress resistance; quantitative trait loci; spectrin beta chain, brain; bZIP transcription factor21/TGA9; leucine-rich repeat family protein; stress defense signaling

\section{Introduction}

Increased solar ultraviolet-B radiation (UV-B, $280-315 \mathrm{~nm}$ ) since the late 1980s is considered a serious environmental issue owing to the lengthy expected time of the recovery of the destroyed stratospheric ozone layer to pre-1980 levels [1,2]. High-intensity UV-B radiation beyond the level of positive stimulus to sessile plants exerts multiple stresses, such as strong light, high temperatures, and dehydration, causing physiological and morphological damages including reduced photosynthetic capacity, leaf discoloration, and reduced biomass and seed yields [3,4]. According to the United Nations Environment Programme (UNEP) annual report, increased UV-B radiation in terrestrial areas reduces plant productivity by about $6 \%$ [5]. To prevent such yield losses in crop plants, genetic studies 
of resistance to high-intensity UV-B radiation as a complex abiotic stress and the identification of genetic elements involved in the UV-B defense response are needed for major crops across the world.

Soybean (Glycine max [L.] Merr.) is one of the most important crops for food, feed, energy production, and industrial resources worldwide. Under supplemental UV-B radiation, soybean cultivars show differences in physiological damage and morphological changes; UV-B-sensitive cultivars show significant yield reductions [6-8]. Quantitative trait loci (QTLs) associated with resistance to supplementary UV-B treatment have been identified on chromosomes (Chrs) 3, 6, 7, and 19 using a recombinant inbred line (RIL) population derived from a cross between Keunol (UV-B sensitive) and Iksan10 (UV-B resistant) [9]. Using the same population, the QTL qUVBT1 on Chr 7 was identified with the 180K AXIOM SoyaSNP array and a gene encoding a UV excision repair protein was identified as a candidate gene involved in UV-B tolerance [10]. In our previous study, we identified the most resistant and most sensitive genotypes to elevated UV-B, Buseok and Cheongja 3, among 140 soybean genotypes, including 94 G. max and 46 G. soja accessions [11]. Transcriptome profiling of these two genotypes differing in UV-B resistance has revealed differentially expressed genes involved in stress defense signaling, immune responses, and reactive oxygen species metabolism [12].

In the present study, we identified QTLs associated with resistance to high-intensity UV-B irradiation using an $\mathrm{F}_{6}$ RIL population of Cheongja 3 (UV-B sensitive) $\times$ Buseok (UV-B resistant). Furthermore, we investigated nucleotide variation in genes located in the QTLs in the mapping parents and their expression levels in response to UV-B treatment.

\section{Results}

\subsection{Phenotypic Evaluation of UV-B Stress Resistance in a RIL Population of Cheongja $3 \times$ Buseok}

UV-B-resistant Buseok and UV-B-sensitive Cheongja 3 showed leaf damage of $26.8 \%$ and $62.4 \%$, respectively, in response to high-intensity UV-B irradiation (Figure 1). The difference in UV-B resistance between the two parents was consistent with the results of previous studies $[11,12]$. The RIL population of Cheongja $3 \times$ Buseok showed high phenotypic variation in leaf damage, ranging from $10 \%$ to $100 \%$, with a mean value of $50.3 \%$ (Figure 1). The normal distribution of the degree of UV-B leaf damage in the RIL population (Shapiro-Wilk: $\mathrm{W}=0.993, \mathrm{P}=0.665$ ), with transgressive segregation, indicates that UV-B resistance is quantitatively regulated by multiple genes.

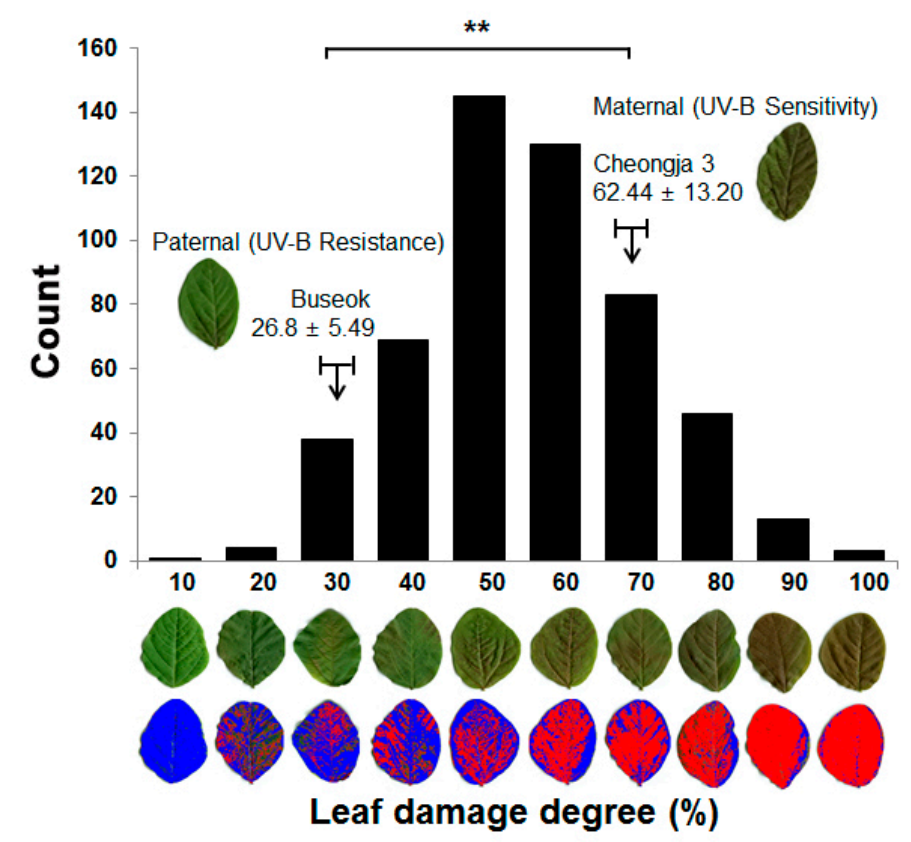

Figure 1. Distribution of the degree of leaf damage under high-intensity ultraviolet-B (UV-B) irradiation in the recombinant inbred line (RIL) population of Cheongja $3 \times$ Buseok. X-axis indicates the degree of 
leaf damage from $10 \%$ to $100 \%$. For each degree of damage, RGB (red-green-blue) and two-color-transformed images of a representative injured leaf are shown. Blue and red in the two-color-transformed image indicate intact and damaged parts of the leaf exposed to UV-B irradiation, respectively. ** indicates a significant difference between Cheongja 3 and Buseok at $p<0.01$ based on a Student's $t$-test.

\subsection{Genotyping-by-Sequencing Analysis and Genetic Linkage Map Construction}

Out of about 562 million genotyping-by-sequencing (GBS) reads generated from two libraries of the two parents and 174 RILs, $441,142,257$ (78.4\%) high-quality clean reads were obtained (Table S1). The number of reads per line ranged from $1,147,211$ to $10,657,712$ with an average of $3,197,053$ reads. From these reads, we identified 271,254 unfiltered single nucleotide polymorphisms (SNPs) differentiating Cheongja 3 and Buseok. We used 4604 high-quality single nucleotide polymorphisms after filtering to construct a genetic linkage map of the RIL population. The final linkage map contained 3136 SNP markers evenly distributed on 20 chromosomes according to their physical locations on the soybean reference genome (Figure S1 and Table S2). The map spanned $3607.3 \mathrm{cM}$ with an average interval of $1.15 \mathrm{cM}$ between adjacent markers (Table S2). On average, each chromosome contained 156 markers spanning an average length of $180.4 \mathrm{cM}$. The chromosomes ranged from $65.8 \mathrm{cM}(\mathrm{Chr} 16)$ to $269.2 \mathrm{cM}$ (Chr 15). The shortest chromosome, Chr 16, was most saturated, containing 95 SNP markers with an average marker density of $0.7 \mathrm{cM}$. Chr 4 had the largest intervals of $2.3 \mathrm{cM}$ between adjacent markers. The longest chromosome, $\mathrm{Chr} 15$, harbored 319 markers, covering $269.2 \mathrm{cM}$ with an average marker interval of only $0.8 \mathrm{cM}$.

\subsection{Identification of QTLs for UV-B Resistance}

Based on the constructed genetic map, we identified four QTLs underlying UV-B resistance in soybean on Chr 12, 14, 10, and 6 (in order of logarithm of odds [LOD] score), together explaining 20.1\% of phenotypic variation (Table 1). The QTL UVBR12-1 was located at Chr12:7,261,406 . . 7,273,570 and had the highest LOD score of 3.8, explaining 9.5\% of the phenotypic variation. The QTLs UVBR14-1 and UVBR10-1 on Chr 14 and 16 had LOD scores of 2.2 and 1.1 and explained $5.3 \%$ and $2.7 \%$ of phenotypic variation, respectively. On Chr 6, a minor QTL for UV-B resistance between markers Chr06:50,843,417 and Chr06:50,873,593 had the lowest $R^{2}$ value (2.6\%) among all QTLs detected (Table 1 ).

We searched for genes located within the four QTLs UVBR6-1, 10-1, 12-1, and 14-1 according to the physical locations of SNP markers associated with the QTLs (Table 1 and Figure 2). Two genes encoding spectrin beta chain, brain (SPTBN, Glyma.12g088600) and bZIP transcription factor21 (bZIP TF21/TGA9, Glyma.12g088700) were located within UVBR12-1 of the $121.6 \mathrm{~kb}$ region flanked by the marker positions Chr12:7273570 and Chr12:72761406 (Table 1 and Figure 2). UVBR6-1 harbored five protein-coding genes anchored in the $30.2 \mathrm{~kb}$ region between Chr06:50,843,417 and Chr06:50,873,593 (Table 1 and Figure 2), including two genes (Glyma.06g319600 and Glyma.06g319700) encoding leucine-rich repeat (LRR) family proteins, one gene (Glyma.06g319800) for alfin-like 1, and two genes (Glyma.06g319900 and Glyma.06g320000) encoding family with sequence similarity 136, member A (FAM136A)-like protein (Table 1 and Figure 2). The 1.33 Mb genomic region of UVBR10-1 (Chr10:41,185,273 ... Chr10:42,517,624) included 140 genes, and 60 genes were located in the $647.6 \mathrm{~kb}$ genomic region corresponding to UVBR14-1 (Chr14:47,368,499 ... Chr14:46,720,930) (Table 1 and Table S3). 
Table 1. Quantitative trait loci (QTLs) for UV-B stress resistance identified by inclusive composite interval mapping in 176 RILs derived from Cheongja $3 \times$ Buseok.

\begin{tabular}{|c|c|c|c|c|c|c|c|c|}
\hline Locus & Left Marker & Right Marker & Position $^{\text {a }}$ (cM) & LOD $^{b}$ & Add $^{c}$ & $R^{2 \mathrm{~d}}(\%)$ & No. of Genes e & Known Stress-Related QTLs \\
\hline UVBR12-1 & Chr12:7261406 & Chr12:7273570 & 74 & 3.8 & -0.4 & 9.5 & 2 & $\begin{array}{c}\text { Drought tolerance 6-4 } \\
\text { SCN 39-4 }\end{array}$ \\
\hline UVBR14-1 & Chr14:46720930 & Chr14:47368499 & 189 & 2.2 & 0.3 & 5.3 & 60 & $\begin{array}{c}\text { Fe-effect 3-2, 9-2, 10-3 } \\
\text { Flood tolerance 4-7 }\end{array}$ \\
\hline UVBR10-1 & Chr10:41185273 & Chr10:42517624 & 160 & 1.1 & -0.2 & 2.7 & 142 & $\begin{array}{l}\text { Drought tolerance 6-3 } \\
\text { Sclero 2-23, 4-10, 3-18 } \\
\text { Phytoph 5-3 }\end{array}$ \\
\hline UVBR6-1 & Chr06:50843417 & Chr06:50873593 & 38 & 1.1 & 0.2 & 2.6 & 5 & Plant damage, UV-B induced 1-2 \\
\hline
\end{tabular}

a Genetic position of a QTL peak in the linkage map constructed in the present study. ${ }^{\mathrm{b}}$ Maximum-likelihood logarithm of odds (LOD) score for the individual QTL. ${ }^{\mathrm{c}}$ Allelic effect. $\mathrm{d}$ Percent of phenotypic variance explained by the QTL. ${ }^{\mathrm{e}}$ Number of protein-coding genes within marker intervals on the basis of G. max gene models ver. 1.1. ${ }^{\mathrm{f}}$ Known stress-related QTLs within $2 \mathrm{Mb}$ surrounding the QTLs identified in this study. 


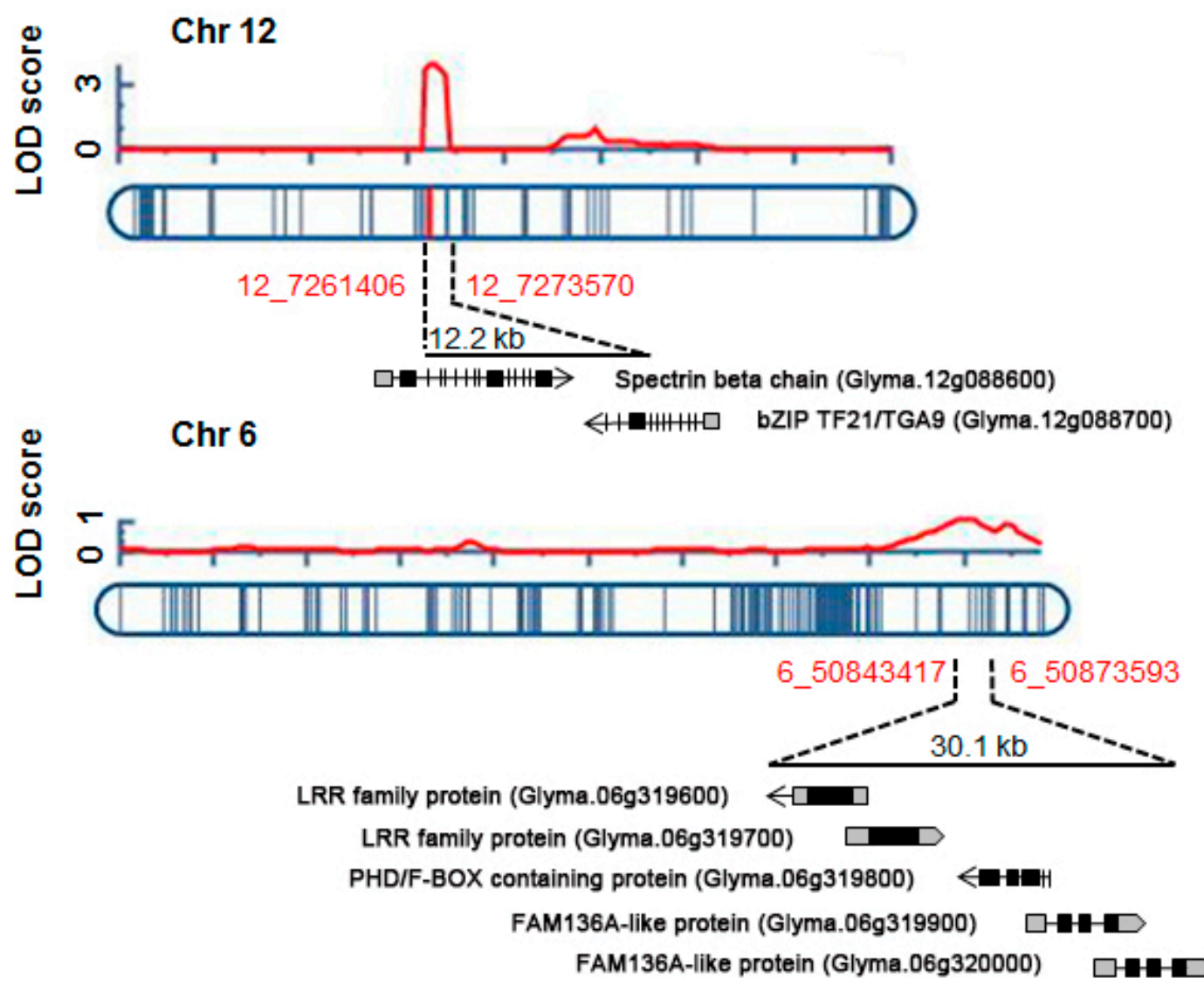

Figure 2. LOD peaks and chromosomal locations of two QTLs UVBR12-1 and UVBR6-1 for resistance to high-intensity UV-B irradiation on chromosome (Chr) 12 and Chr 6. Red curves present LOD score distribution of detected QTLs on Chr 12 and Chr 6. These loci contain two and five protein-coding genes, respectively.

\subsection{SNPs and Gene Expression Differences in the QTLs in the Mapping Parents}

To identify candidate genes in the QTLs associated with UV-B resistance, we investigated SNPs in two and five genes within the two UV-B resistance QTLs UVBR12-1 and UVBR6-1, respectively, by a sequence analysis of the parental genotypes Cheongja 3 and Buseok (Table). In UVBR12-1, the two genes SPTBN (Glyma.12G088600) and bZIP TF21/TGA9 (Glyma.12G088700) had nine and 25 SNPs between Cheongja 3 and Buseok, respectively (Table S4). In SPTBN (Glyma.12G088600), we detected one and five SNPs in the up- and downstream regions, respectively. We found three additional SNPs in the genic regions of SPTBN, one of which was a synonymous SNP in an exon (Figure 3). Among 25 total SNPs in bZIP TF21/TGA9 (Glyma.12G088700), we found 15 SNPs in the genic region, consisting of three in $3^{\prime} U T R, 10$ in introns and two in exons (Figure 3 and Table S5). Each of the $2 \mathrm{~kb}$ up- and downstream regions of $b Z I P$ TF21/TGA9 possessed five SNPs. Two SNPs in the coding sequence of bZIP TF21/TGA9 were synonymous (Figure 3).

Among five genes within UVBR6-1, three genes had nucleotide differences between Cheongja 3 and Buseok (Tables S4 and S5). In Glyma.06g319700, encoding a LRR family protein, we detected two nonsynonymous missense mutations in exons and two SNPs in the $2 \mathrm{~kb}$ upstream region (Figure 3). In particular, we discovered amino acid changes from Phe to Leu and Arg to Gly at positions 247 and 254 of Glyma.06g319700, respectively. The two genes Glyma.06g319900 and Glyma.06g320000 (encoding FAM136A-like proteins) had eight and five SNPs in non-coding regions, respectively (Table S5). 
Among five genes within UVBR6-1, three genes had nucleotide differences between Cheongja 3 and Buseok (Tables S4 and S5). In Glyma.06g319700, encoding a LRR family protein, we detected two nonsynonymous missense mutations in exons and two SNPs in the $2 \mathrm{~kb}$ upstream region (Figure 3). In particular, we discovered amino acid changes from Phe to Leu and Arg to Gly at positions 247 and 254 of Glyma.06g319700, respectively. The two genes Glyma.06g319900 and Glyma.06g320000 (encoding FAM136A-like proteins) had eight and five SNPs in non-coding regions, respectively (Table S5).

We further investigated transcriptional differences of SPTBN (Glyma.12G088600) and bZIP TF21/TGA9 (Glyma.12G08870) on UVBR12-1 between Cheongja 3 and Buseok by qRT-PCR (Figure 4) owing to the lack of protein sequence differences between the mapping parents (Figure 3). Both of the genes were upregulated in Buseok subjected to $6 \mathrm{~h}$ of UV-B treatment compared with levels in the control. Relative to UV-B-sensitive Cheongja 3, Buseok showed significantly higher expression levels of the two genes in response to $6 \mathrm{~h}$ of UV-B exposure.

\subsection{Comparisons of UV-B Stress Resistance QTLs with Known Stress-Related QTLs Based on Synteny}

The QTL with the highest LOD, UVBR12-1, on Chr 12 was located near SCN39-4, a QTL for the reaction to the soybean cyst nematode (SCN; Heterodera glycines) linked to Sctt009 (Table 1). The genomic region (Chr12:6,657,383 ... 11,713,748) harboring UVBR12-1 and SCN39-4 had a duplicated block on the same chromosome (Chr12:34,009,158 . . 36,919,294), carrying QTLs for drought and flood tolerance (Drought tolerance 6-4 and Flood tolerance 7-1, respectively) linked to Sat 175 (Figure 5). Three homeologous blocks that show syntenic relationships with the two duplicated regions on Chr 12 were also detected on Chr 6, 11, and 13. The homeologous region (Chr11:24,411,218 . . 26,359,492) on Chr 11 with a median Ks value of 0.135 probably resulted from the recent whole genome duplication (WGD) event 13 million years ago [13] and only had collinearity with the beginning of the genomic region (including only UVBR12-1) on Chr 12. This syntenic block contained six QTLs for SCN resistance, SCN 17-1, 18-2, 20-1, 23-1, 24-1, and 32-2, associated with Satt583. On Chr 13, the marker Satt554, associated with the QTL Asian soybean rust 2-3 for resistance to Asian soybean rust, resided in another duplicated region (Chr13:39,150,251 ... 41,460,183) resulting from the recent WGD (median Ks, 0.133). In UVBR12-1, bZIP TF21/TGA9 was retained in all three duplicated regions but SPTBN was only retained in the homeologous block on Chr 11 and was lost in the other blocks (Figure 5). On Chr 6, the remaining duplicated block (Chr06:47,897,433 ... 50,898,694) was mainly conserved with the middle part of the genomic region of UVBR12-1. This block with the UV-B resistance QTL UVBR6-1 included another UV-B-related QTL (Plant damage UV-B induced 1-2) as well as biotic stress-related QTLs, including SCN 17-3 and 20-2, and SDS 7-5 for sudden death syndrome resistance (reaction to Fusarium solani f. sp. glycines), which were associated with Satt371.

The UV-B resistance QTL UVR14-1 on Chr 14 was mapped near three QTLs for iron efficiency, Fe-effect 3-2, 9-2, and 10-3 (Table 1). On Chr 10, UVR10-1 was co-localized with several QTLs related to biotic as well as abiotic stresses, including Flood tolerance 4-7, Drought tolerance 6-3, Sclero (Reaction to Sclerotinia sclerotiorum infection) 2-23, 4-10, and 3-18, and Phytoph (Reaction to Phytophthora sojae infection) 5-3. 

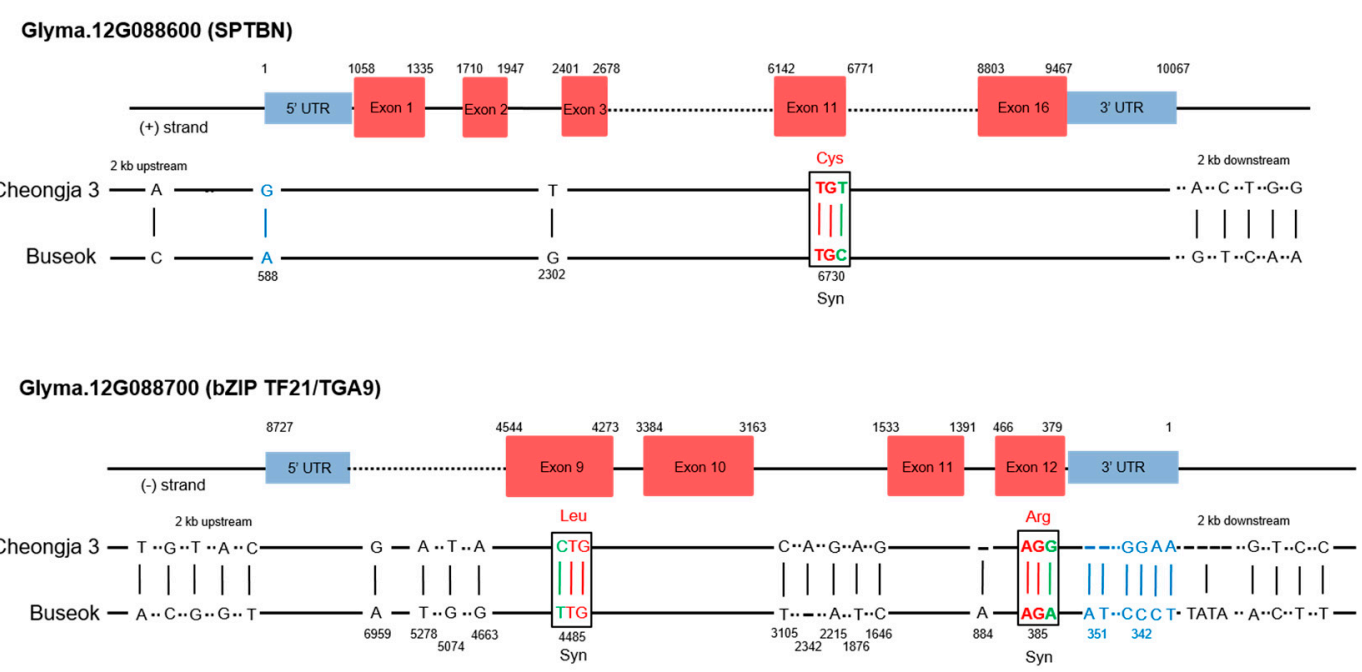

Glyma.06G319700 (LRR family protein)

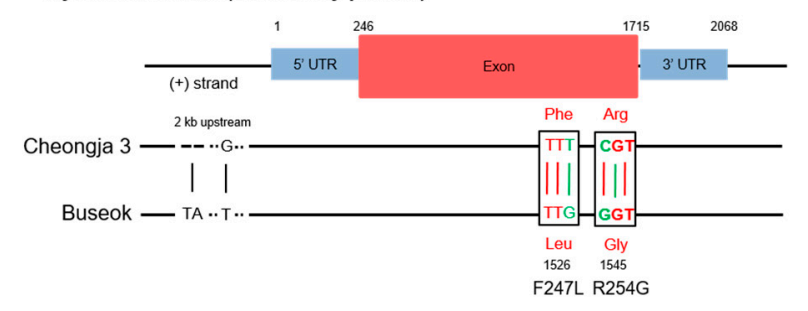

Figure 3. Structures of three genes encoding SPTBN (Glyma.12G088600), bZIP TF21/TGA9 (Glyma.12G088700), and LRR family protein (Glyma.06G319700), and single nucleotide polymorphisms (SNPs) in the mapping parents Cheongja and Buseok. Exons and untranslated regions (UTRs) are indicated by red- and blue-filled boxes, respectively. SNP positions, nucleotide replacements, and amino acid substitutions between Cheongja 3 and Buseok are presented. One and two SNPs in exons of SPTBN (Glyma.12G088600) and bZIP TF21/TGA9 (Glyma.12G088700), respectively, were synonymous. The SNPs T/G and C/G in exons of Glyma.06G319700 (LRR family protein) caused amino acid replacements of Phe to Leu and Arg to Gly at the 247th and 254th residues, respectively.
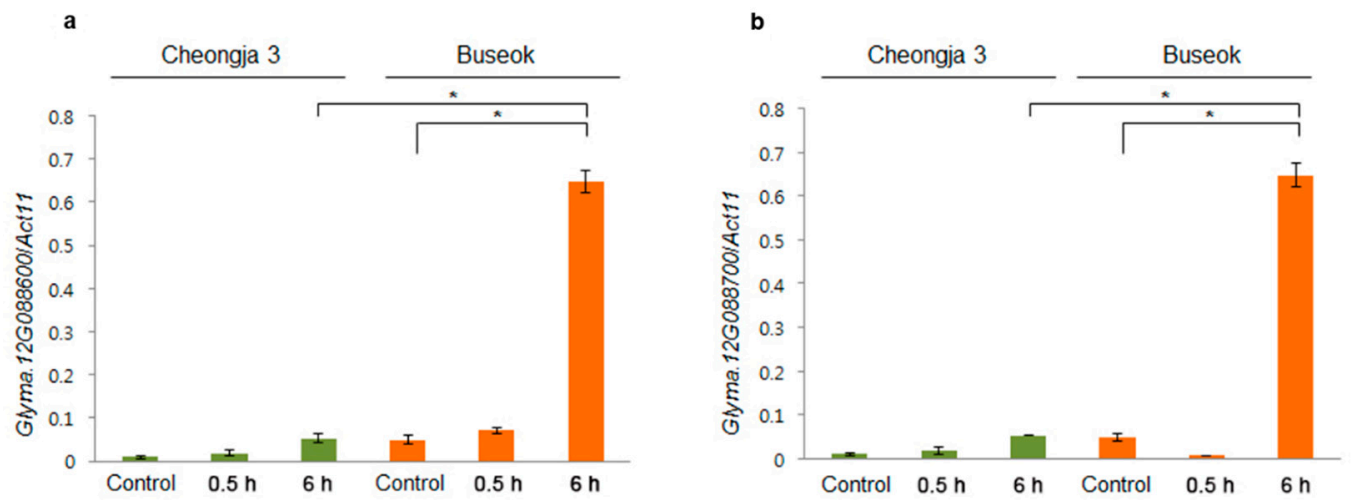

Figure 4. Expression levels of SPTBN (Glyma.12G088600) (a) and bZIP TF21/TGA9 (Glyma.12G088700) (b) in Cheongja 3 (green) and Buseok (orange). Y-axis represents the relative transcript abundance determined by qRT-PCR. Control, 0.5 , and $6 \mathrm{~h}$ on the X-axis refer to $0,0.5$, and $6 \mathrm{~h}$ UV-B irradiation, respectively. Error bars represent the standard error for three independent replicates. ${ }^{*}$, significant at $p<0.05$. 


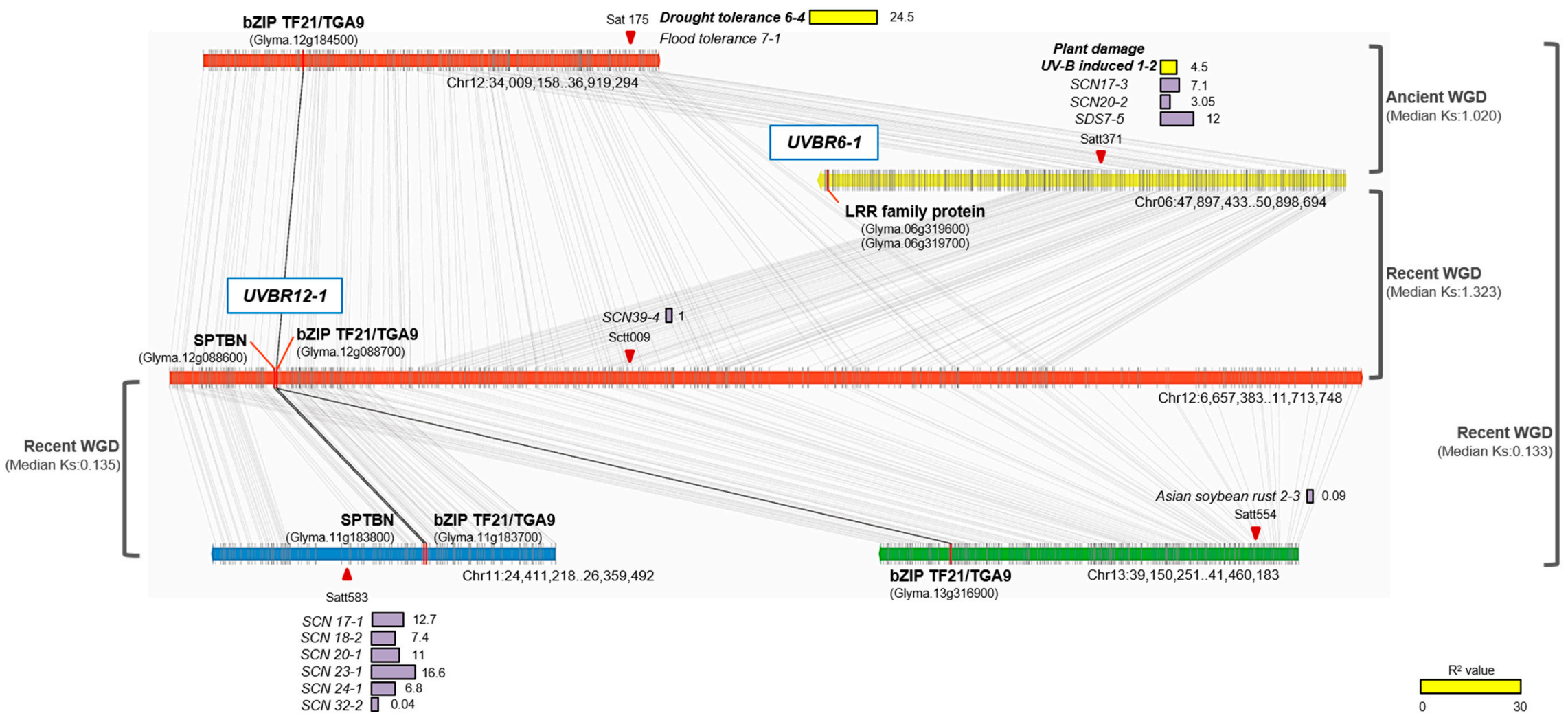

Figure 5. Syntenic conservation of two genomic regions of UVBR12-1 and UVBR6-1 and comparisons with other known stress-related QTL regions. The genomic region with UVBR12-1 (Chr12:6,657,383 ... 11,713,748) had a duplicated block on the same Chr 12 (Chr12:34,009,158...36,919,294) and three additional homeologous regions on Chr 6, 11, and 13. Whole genome duplication (WGD) events producing each pair of syntenic blocks are defined as recent or ancient based on median Ks values. Among two genes in UVBR12-1, bZIP TF21/TGA9 was retained in all duplicated regions but SPTBN was only retained in the homeologous block on Chr 11 and was lost in the other blocks. Yellow and purple bars indicate phenotypic R2 values of known QTLs related to abiotic and biotic stress, respectively. SPTBN, spectra beta chain, brain; bZIP TF21/TGA9, bZIP transcription factor 21/TGACG motif-binding 9; SCN, Reaction to Heterodera glycines; SDS, Reaction to Fusarium solani f. sp. glycines infection. 


\section{Discussion}

Crop plants under abiotic stresses, such as drought, salinity, and extreme temperatures, induce common cellular signaling mechanisms associated with osmotic stress, which disrupts homeostasis and alters the ion balance in the cell [14-16]. Similarly, high-intensity UV-B radiation above ambient levels induces cellular responses to nonspecific (genotoxic) damage, similar to various stress defense mechanisms but distinct from photomorphogenesis to low-dose UV-B, indicating that it is a complex environmental stressor [17-22]. We previously found that, compared with gene expression in UV-B-sensitive Cheongja 3, UV-B-resistant Buseok exhibits the upregulation of genes involved in phosphatidic acid-dependent signaling pathways as well as several downstream pathways, such as ABA signaling, mitogen-activated protein kinase cascades, and reactive oxygen species overproduction [12]. Therefore, high-density UV-B irradiation is a useful stress treatment for molecular biological and genetic studies of genes involved in extensive resistance to multiple abiotic stresses caused by climate change.

To identify the genetic elements responsible for UV-B resistance by QTL mapping, we developed a RIL population from a cross between UV-B-sensitive Cheongja 3 and UV-B-resistant Buseok. High-intensity UV-B irradiation turns most leaves yellow with red spots in UV-B-sensitive Cheongja 3, resulting in a dramatic loss in aerial dry weight, whereas Buseok retains more healthy leaves $[11,12]$. Our RIL population showed a wide distribution in the degree of leaf damage caused by UV-B stress (Figure 1). We identified four QTLs associated with UV-B resistance on Chr 12, 14, 10, and 6 (in order of LOD score) in this population, and all LOD scores were less than four (Table 1). Using a different UV-B-resistant soybean genotype, Iksan 10, previous QTL studies based on SSR genotyping and a SoyaSNP assay identified two major loci for UV-B resistance on Chr 19 and 7 , respectively $[9,10]$. These previously reported QTLs do not overlap with our UV-B resistance QTLs. We identified a novel QTL, UVBR12-1, with a LOD score of 3.76 on Chr 12, explaining about $10 \%$ of phenotypic variation (Table 1). Thus, the genetic determinants of UV-B resistance in the two soybean genotypes Buseok and Iksan 10 are presumed to be different. However, a minor QTL, UVBR6-1, on Chr 6 was mapped in the vicinity of a known minor QTL UV-B induced plant damage 1-2 associated with Satt371 [9] (Table 1 and Figure 5), which was only detected by multiple regression in the previous study [9].

The newly identified UV-B resistance QTLs were co-localized with other known QTLs for resistance to biotic as well as abiotic stresses (Table 1 and Figure 5). These biotic stresses include infections with $H$. glycines, F. solani f. sp. glycines, S. sclerotiorum, and P. sojae, and the abiotic stresses include drought, flood, and iron deficiency. Plants subjected to different combinations of multiple stresses show extensive overlap and crosstalk between stress-response signaling pathways, together with specific responses to individual stresses, indicating a high degree of complexity in plant molecular responses to external stresses [23]. Our results indicate that some genetic elements mediating resistance to UV-B stress may be shared with mechanisms underlying responses to other stresses, consistent with previous findings [12,24].

We identified candidate genes controlling resistance to high-intensity UV-B irradiation among two genes on UVBR12-1 and six genes on UVBR6-1 (Figures 3 and 4). The gene Glyma.12G088600 on UVBR12-1 is a homolog of Arabidopsis SPTBN (AT5G22450), likely encoding a $\beta$-spectrin ( $\beta$ I to $\beta V)$, which are actin-binding proteins in mammals [25]. Though plants, including Arabidopsis, lack spectrins or spectrin-like proteins [26,27], sequences with spectrin repeats and N-terminal calponin homology domains for actin binding are present in the Arabidopsis genome [28]. Additionally, spectrins or spectrin-like proteins are localized in plant cellular organelles, such as the Golgi apparatus [29], endoplasmic reticulum [30], and nucleus [31], and in the border plasma membrane of elongating plant cells $[32,33]$. In mammalian cells, actins bound to $\beta$-spectrins and other actin-binding proteins, such as Protein 4.1, Adducin, and Dematin, are connected to the junctional complex at the intracellular side of the plasma membrane [34-36]; these are implicated in signal targeting as well as the maintenance of cell shape and structure $[25,36]$. $\beta$-Spectrins interact with membrane phosphoinositides (PtdIns) via Pleckstrin homology $(\mathrm{PH})$ domains present in a number of proteins involved in cellular signaling [37-39]. Direct evidence for a relationship between the spectrin-based membrane cytoskeleton and plant stress 
signaling is very limited; however, levels of PtdIns, such as PtdIns4P and PtdIns(4,5)P2, and other phospholipid molecules in plant cells are increased by osmotic stress from salinity and dehydration [40]. Several genes encoding phosphatidylinositol phosphate kinases and phosphatidylinositol-specific phospholipase $C$ involved in phospholipid signaling are upregulated in Buseok after exposure to high-intensity UV-B [12]. In this study, despite a lack of amino acid differences between the mapping parents (Figure 4 and Table S6), elevated SPTBN expression in response to UV-B stress was observed in Buseok but not in Cheongja 3 (Figure 4). Further investigations are necessary to characterize the cellular function of SPTBN in soybean using knockout and overexpression mutants.

HY5 (elongated hypocotyl 5) and HYH (HY5 homolog) are bZIP TFs induced by UV-B. They are key components of low-level UV-B photomorphogenic signaling mediated by $\beta$-propeller protein and E3 ubiquitin ligase, encoded by UVR8 (UV Resistance Locus 8) and COP1 (Constitutively Photomorphogenic 1), respectively [19,41,42]. The bZIP TF gene Glyma.12g088700 on UVBR12-1 is an ortholog of Arabidopsis bZIP21/TGA9 (AT1G08320), which belongs to Clade IV of the TGACG motif-binding (TGA) protein family and is essential for anther development $[43,44]$. In a phylogenetic tree of bZIP genes from G. max and A. thaliana, the bZIP21/TGA9 orthologs Glyma.12g088700 and AT1G08320 belong to a different cluster from that including HY5 (AT5g11260) (Figure S2), corresponding to previous classifications in A. thaliana [43]. The expression of Glyma.12g088700 on UVR12-1 was significantly increased by exposure to high-intensity UV-B in Buseok (Figure 4), but direct evidence for the role of this type of bZIP gene in resistance to abiotic stresses is lacking. bZIP21/TGA9 and TGA10 are only known to be involved in plant pathogen-associated molecular pattern-triggered immunity stimulated by the immunogenic peptide of bacterial flagellin (flg22) $[45,46]$.

In UVBR6-1, Glyma.06g319700 showed amino acid differences between the mapping parents; the gene is orthologous to the Arabidopsis gene AT1G33590, which encodes a LRR family protein. The LRR motif plays a central role in recognizing different pathogen-associated molecules in the innate host defense of plants and animals [47]. Recent studies have shown that several genes encoding LRR-containing proteins are involved in the abiotic stress response [48], including LP2 (LEAF PANICLE 2), TaPRK2697 (Triticum aestivum PROTEIN OF RECEPTOR KINASES 26697), AtPXL1 (Arabidopsis thaliana PHLOEM INTERCALATED WITH XYLEM-LIKE 1), and LRR-RLK-VIII (LEUCINE-RICH REPEAT RECEPTOR-LIKE KINASE VIII), which are upregulated by drought, salt, cold, and toxic metals, respectively, in diverse plant species [49-52].

Three candidate genes responsible for UV-B resistance in soybean were identified from two QTLs, UVBR12-1 and UVBR6-1. These genes encode SPTBN, bZIP TF21/TGA9, and a LRR family protein and are likely involved in stress defense signaling; they should be experimentally verified using overexpression or knockout mutants. Further functional studies will improve our understanding of UV-B stress defense mechanisms. Furthermore, our results provide powerful genetic tools for more efficient and precise breeding programs aimed at the development of highly adaptable soybean cultivars under various abiotic stresses caused by global climate change.

\section{Materials and Methods}

\subsection{Plant Materials and DNA Extraction}

Two soybean genotypes were used as parents to develop a RIL population for genetic map construction and the QTL analysis. Buseok, a paternal line, is a UV-B-resistant genotype, and Cheongja 3, a maternal line, is a UV-B-sensitive genotype [11,12]. Artificial crossing was performed in summer 2012, and $176 \mathrm{~F}_{6}$ RILs were generated from $\mathrm{F}_{2}$ seeds using the single seed descent method from winter 2012 to spring 2016. Healthy young leaves from two parental genotypes and RILs were collected, and high-quality genomic DNA was extracted using the GeneAll ${ }^{\circledR}$ Exgene Plant SV Kit (GeneAll Biotechnology, Seoul, Korea). DNA quality was assessed by the 260/280 nm ratio using a Nanodrop 3000 spectrometer (Thermo Scientific, Wilmington, DE, USA). DNA was quantified using 
the Invitrogen Quant-iT PicoGreen ${ }^{\circledR}$ dsDNA Assay Kit (Life Technologies, Burlington, ON, Canada) and adjusted to $20 \mathrm{ng} / \mu \mathrm{L}$.

\subsection{UV-B Treatment and Phenotypic Evaluation}

Soybean seeds of the mapping parents and their RILs were planted (three seeds per pot) in 3 L pots containing a 1:1 mixture of desalinated sand and commercial potting soil (Baroker, Seoul Bio Co., Seoul, Korea) in a greenhouse at the experimental farm of Seoul National University, Suwon, Korea in August 2016. Supplemental UV-B irradiation was used to treat soybean plants at the V3 growth stage two to three weeks after emergence according to a previous study [11]. Supplemental UV-B radiation was applied using G40T10E UV-B lamps (Sankyo Denki, Nagano, Japan) with a mean UV-B intensity of $5.68 \pm 0.4 \mathrm{Wm}^{-2}$ at the plant level under the lamps. The plants were exposed to high-intensity UV-B stress for $1 \mathrm{~h}$ every day at 11:00 am from 17 August to 20 August. The intensity of $1 \mathrm{~h}$ of UV-B irradiation was equivalent to two times $11.5 \mathrm{~kJ} / \mathrm{m}^{2}$, the daily soybean UV-B biological effective dose $[12,53]$. Three sets of first and second trifoliate leaves above unifoliate leaves from three plants after UV-B treatment were collected with three replications per line. To investigate leaf color changes caused by high-intensity UV-B exposure, the collected leaf samples were scanned with an EPSON Perfection V33 scanner (Epson Inc., Long Beach, CA, USA) and scanned images were analyzed using the WinDIAS 3 Leaf Image Analysis System (DELTA-T DEVICES LTD., Cambridge, UK). The degree of damage was scored on a scale of $1-10$ (where $1=0 \%-10 \%, 2=11 \%-20 \%$, and up to $10=$ $91 \%-100 \%$; damaged area/healthy area $\times 100(\%))$ and these scores was used in QTL mapping for UV-B resistance. Phenotypic distribution was tested for deviation from normality with the Shapiro-Wilk test.

\subsection{Genotyping-by-Sequencing}

A GBS technique was used to detect SNPs to genotype the RIL population. A GBS library was constructed following the protocol described by [54]. DNAs from the mapping parents and 176 RILs were digested individually with ApeKI, which recognizes a degenerate 5 bp sequence (GCWGC, where $\mathrm{W}$ is either $\mathrm{A}$ or $\mathrm{T}$ ). Barcoded adapters were ligated with digested DNA fragments (Table S6), and ligated DNA was amplified with appropriate primers. Then, two separate libraries were constructed by pooling amplified DNA samples of 88 RILs for each library. Single-end sequencing of the GBS libraries was performed on two lanes of an Illumina HiSeq2000 instrument (Illumina Inc., San Diego, CA, USA).

\subsection{Sequence Analysis, Genetic Map Construction, and QTL Analysis}

Raw GBS reads were mapped against the G. max reference genome (Wm82.a2) downloaded from Phytozome (https:/phytozome.jgi.doe.gov/pz/portal.html) using Bowtie v2.1 after sequence quality control, such as the removal of barcode, adapter, and ApeKI overhang sequences as well as reads with Phred scores of $<15$ for at least $80 \%$ of bases $[55,56]$. SNPs were called using in-house python scripts with the following filtering criteria: read depth $\geq 3$, Q value $\geq 30$, and missing error rate $\leq 10 \%$. All SNPs were deposited in The European Variation Archive (https://www.ebi.ac.uk/ eva,PRJEB32685). Using identified SNP markers, a genetic map was constructed with JoinMap 4.1 (https://www.kyazma.nl/index.php/mc.JoinMap). SNP markers were grouped using the Kosambi mapping function, and segregation distortion of individual markers was calculated using the $\chi^{2}$ test in JoinMap 4.1. The SNP genotyping data and degrees of leaf damage by UV-B treatment for 176 RILs of Cheongja $3 \times$ Buseok were evaluated using QTL IciMapping v.4.1.0.0, and QTLs for resistance to high-intensity UV-B were identified by inclusive composite interval mapping (https: //www.integratedbreeding.net/386/breeding-services/more-software-tools/icimapping). To determine the statistically significant threshold for the LOD score, 1000 permutation test was performed at the $5 \%$ significance level. 


\subsection{SNP Survey and qRT-PCR Analysis of Genes in the QTLs for UV-B Stress Resistance}

To investigate SNPs in genes located in the QTLs for UV-B resistance in Cheongja 3 and Buseok, whole genome sequences of these genotypes reported in a previous study were used [11]; the sequences are available from the website of the Crop Genomics Laboratory at Seoul National University (http://plantgenomics.snu.ac.kr/). To detect high-confidence SNPs, the following cut-off values were used: minimum read depth of 5 , maximum read depth of 20, and Phred-scaled probability score above 30. A maximum read depth of 20 was applied to remove false positive SNP calls that may result from duplicated or repetitive sequences. The SNP positions were determined based on the Glyma 2.0 gene models (Wm82.a2.v1). To compare gene expression levels within the QTL UVBR12-1 for UV-B resistance between Cheongja 3 and Buseok, gene-specific primers were designed for qRT-PCR using Primer3 (http://bioinfo.ut.ee/primer3-0.4.0/) (Table S7). Total RNA from each sample for control, $0.5 \mathrm{~h}$, and $6 \mathrm{~h}$ UV-B treatments in Cheongja 3 and Buseok was used to synthesize cDNA using a Bio-Rad iScript ${ }^{\mathrm{TM}}$ cDNA Synthesis Kit (Hercules, CA, USA). UV-B treatments for qRT-PCR analysis followed as described in the previous RNA-seq study [12]: $0.5 \mathrm{~h}$ exposure (equivalent to $11.5 \mathrm{KJ} / \mathrm{m}^{2}$ soybean UV-B $\mathrm{BE}_{\mathrm{BE}}$ ) induced photomorphogenic (non-damaging) cellular response to low-dose UV-B radiation, while $6 \mathrm{~h}$ exposure (12-times higher than daily UV-B $\mathrm{B}_{\mathrm{BE}}$ ) resulted in nonspecific (genotoxic) damage induced by abiotic stress. qRT-PCR was performed using the synthesized cDNA as a template and a Bio-Rad iQ ${ }^{\text {TM }}$ SYBR Green Supermix Kit on a LightCycler ${ }^{\circledR} 480$ (Roche Diagnostics, Laval, QC, Canada). The qRT-PCR mixture in a total volume of $20 \mu \mathrm{L}$ contained $100 \mathrm{ng}$ of cDNA, each primer at $300 \mu \mathrm{M}$, $8 \mu \mathrm{L}$ of sterile water, and $10 \mu \mathrm{L}$ of Bio-Rad iQ ${ }^{\mathrm{TM}}$ SYBR Green Supermix. The amplification conditions were as follows: $5 \mathrm{~min}$ of denaturation at $95{ }^{\circ} \mathrm{C}$ followed by 40 cycles of $95^{\circ} \mathrm{C}$ for $10 \mathrm{~s}$ and $60^{\circ} \mathrm{C}$ for $1 \mathrm{~min}$. The samples were analyzed in triplicate, and actin was used as a reference gene for the normalization of target gene expression in soybean. Data were analyzed based on the stable expression level of the reference gene according to the method of Livak and Schmittgen [57].

\subsection{Co-Localization of Stress-Related QTLs with UV-B Resistance QTLS}

Abiotic and biotic stress-related QTLs for soybean were obtained from the SoyBase website (http://soybase.org/). The chromosomal positions of QTLs on soybean chromosomes were determined using marker information from version 4.0 of the soybean map from SoyBase [58]. Syntenic blocks in the soybean reference genome sequence were analyzed using MCScanX with default parameters [59], and syntenic blocks overlapping with genomic regions of approximately 2 to $5 \mathrm{Mbp}$ encompassing SNP markers associated with UV-B resistance were identified.

Supplementary Materials: Supplementary materials can be found at http://www.mdpi.com/1422-0067/20/13/3287/s1.

Author Contributions: Conceptualization, M.Y.Y. and M.Y.K.; methodology, M.Y.Y. and M.Y.K.; software, M.Y.Y. and T.L.; validation, M.Y.Y.; formal analysis, M.Y.Y.; investigation, M.Y.Y.; resources, M.Y.Y.; data curation, J.H.; writing-original draft preparation, Y.M.Y. and M.Y.K.; writing-review and editing, M.Y.K., J.H., K.D.K and S.-H.L.; visualization, M.Y.Y., T.L. and M.Y.K.; supervision, S.-H.L.; project administration, S.-H.L.; funding acquisition, S.-H.L.

Funding: This work was supported by the Next Generation BioGreen 21 Program (Code No. PJ01322401), Rural Development Administration, Republic of Korea

Conflicts of Interest: The authors declare no conflict of interest.

\section{Abbreviations}

$\begin{array}{ll}\text { UV-B } & \text { Ultraviolet-B } \\ \text { QTL } & \text { Quantitative trait locus } \\ \text { RIL } & \text { Recombinant inbred line } \\ \text { LOD } & \text { Logarithm of odds } \\ \text { GBS } & \text { Genotyping-by-sequencing } \\ \text { SNP } & \text { Single nucleotide polymorphism } \\ \text { WGD } & \text { Whole genome duplication }\end{array}$




$\begin{array}{ll}\text { SCN } & \text { Soybean cyst nematode } \\ \text { SPTBN } & \text { Spectrin beta chain, brain } \\ \text { bZIP TF21/TGA9 } & \text { bZIP transcription factor21/TGACC motif-binding 9 } \\ \text { LRR } & \text { Leucine-rich repeat }\end{array}$

\section{References}

1. World Meteorological Organization, 2008. WMO Statement on the Status of the Global Climate in 2007. WMO-No. 1031. Available online: https://library.wmo.int/doc_num.php?explnum_id=3457 (accessed on 20 February 2019).

2. Petrescu, R.V.; Aversa, R.; Apicella, A.; Petrescu, F.I. NASA sees first in 2018 the direct proof of ozone hole recovery. J. Aircr. Spacecr. Technolog. 2018, 2, 53-64. [CrossRef]

3. Rozema, J.; van de Staaij, J.; Björn, L.O.; Caldwell, M. UV-B as an environmental factor in plant life: Stress and regulation. Trends Ecol. Evolut. 1997, 12, 22-28. [CrossRef]

4. Frohnmeyer, H.; Staiger, D. Ultraviolet-B radiation-mediated responses in plants. Balancing damage and protection. Plant Physiol. 2003, 133, 1420-1428. [CrossRef] [PubMed]

5. UNEP. 2010 Annual Report. 2011. Available online: https://wedocs.unep.org/handle/20.500.11822/7915 (accessed on 20 February 2019).

6. Murali, N.; Teramura, A.H.; Randall, S.K. Response differences between two soybean cultivars with contrasting UV-B radiation sensitivities. Photochem. Photobiol. 1988, 48, 653-657. [CrossRef]

7. Teramura, A.H.; Sullivan, J.H.; Lydon, J. Effects of UV-B radiation on soybean yield and seed quality: A 6-year field study. Physiol. Plantarum. 1990, 80, 5-11. [CrossRef]

8. Baroniya, S.S.; Kataria, S.; Pandey, G.P.; Guruprasad, K.N. Intraspecific variation in sensitivity to ambient ultraviolet-B radiation in growth and yield characteristics of eight soybean cultivars grown under field conditions. Braz. J. Plant Physiol. 2011, 23, 197-202. [CrossRef]

9. Shim, H.C.; Ha, B.K.; Yoo, M.; Kang, S.T. Detection of quantitative trait loci controlling UV-B resistance in soybean. Euphytica 2015, 202, 109-118. [CrossRef]

10. Lee, J.S.; Kim, S.; Ha, B.K.; Kang, S.T. Positional mapping and identification of novel quantitative trait locus responsible for UV-B radiation tolerance in soybean (Glycine max [L.] Merr.). Mol. Breed. 2016, 36, 1-10. [CrossRef]

11. Kim, K.D.; Yun, M.Y.; Shin, J.H.; Kang, Y.J.; Kim, M.Y.; Lee, S.H. Underlying genetic variation in the response of cultivated and wild soybean to enhanced ultraviolet-B radiation. Euphytica 2015, 202, 207-217. [CrossRef]

12. Yoon, M.Y.; Kim, M.Y.; Shim, S.; Kim, K.D.; Ha, J.; Shin, J.H.; Lee, S.H. Transcriptomic profiling of soybean in response to high-intensity UV-B irradiation reveals stress defense signaling. Front. Plant Sci. 2016, 7, 1917. [CrossRef]

13. Schmutz, J.; Cannon, S.B.; Schlueter, J.; Ma, J.; Mitros, T.; Nelson, W.; Hyten, D.L.; Song, Q.; Thelen, J.J.; Cheng, J.; et al. Genome sequence of the palaeopolyploid soybean. Nature 2010, 463, 178-183. [CrossRef] [PubMed]

14. Wang, W.; Vinocur, B.; Altman, A. Plant responses to drought, salinity and extreme temperatures: Towards genetic engineering for stress tolerance. Planta 2003, 218, 1-14. [CrossRef] [PubMed]

15. Shinozaki, K.; Yamaguchi-Shinozaki, K. Molecular responses to dehydration and low temperature: Differences and cross-talk between two stress signaling pathways. Curr. Opin. Plant Biol. 2000, 3, 217-223. [CrossRef]

16. Knight, H.; Knight, M.R. Abiotic stress signaling pathways: Specificity and cross-talk. Trends Plant Sci. 2001, 6, 262-267. [CrossRef]

17. Jordan, B.R. Review: Molecular response of plant cells to UV-B stress. Funct. Plant Biol. 2002, 29 , 909-916. [CrossRef]

18. Paul, N.D.; Gwynn-Jones, D. Ecological roles of solar UV radiation: Towards an integrated approach. Trends Ecol. Evolut. 2003, 18, 48-55. [CrossRef]

19. Jenkins, G.I. Signal transduction in responses to UV-B radiation. Annu. Rev. Plant Biol. 2009, 60, 407-431. [CrossRef]

20. Heijde, M.; Ulm, R. Reversion of the Arabidopsis UV-B photoreceptor UVR8 to the homodimeric ground state. Proc. Natl. Acad. Sci. USA 2013, 110, 1113-1118. [CrossRef] 
21. Müller-Xing, R.; Xing, Q.; Goodrich, J. Footprints of the sun: Memory of UV and light stress in plants. Front. Plant Sci. 2014, 5, 474. [CrossRef]

22. Parihar, P.; Singh, S.; Singh, R.; Singh, V.P.; Prasad, S.M. Changing scenario in plant UV-B research: UV-B from a generic stressor to a specific regulator. J. Photochem. Photobiol. B Biol. 2015, 153, 334-343. [CrossRef]

23. Suzuki, N.; Rivero, R.M.; Shulaev, V.; Blumwald, E.; Mittler, R. Abiotic and biotic stress combinations. New Phytol. 2014, 203, 32-43. [CrossRef] [PubMed]

24. Yoon, M.Y.; Kim, M.Y.; Lee, J.; Lee, T.; Kim, K.H.; Ha, J.; Kim, Y.H.; Lee, S.H. Transcriptomic profiling of soybean in response to UV-B and Xanthomonas axonopodis treatment reveals shared gene components in stress defense pathways. Genes Genom. 2017, 39, 225-236. [CrossRef]

25. Machnicka, B.; Czogalla, A.; Hryniewicz-Jankowska, A.; Bogusławska, D.M.; Grochowalska, R.; Heger, E.; Sikorski, A.F. Spectrins: A structural platform for stabilization and activation of membrane channels, receptors and transporters. Biochim. Biophys. Acta Biomembr 2014, 1838, 620-634. [CrossRef] [PubMed]

26. Bennett, V.; Baines, A.J. Spectrin and ankyrin-based pathways: Metazoan inventions for integrating cells into tissues. Physiol. Rev. 2001, 81, 1353-1392. [CrossRef] [PubMed]

27. Drøbak, B.K.; Franklin-Tong, V.E.; Staiger, C.J. The role of the actin cytoskeleton in plant cell signaling. New Phytol. 2004, 163, 13-30. [CrossRef]

28. Young, K.G.; Kothary, R. Spectrin repeat proteins in the nucleus. BioEssays 2005, 2 7, 144-152. [CrossRef]

29. Holzinger, A.; De Ruijter, N.; Emons, A.M.; LüTz-Meindl, U. Spectrin-like proteins in green algae (Desmidiaceae). Cell Biol. Int. 1999, 23, 335-344. [CrossRef]

30. Braun, M. Association of spectrin-like proteins with the actin-organized aggregate of endoplasmic reticulum in the spitzenkörper of gravitropically tip-growing plant cells. Plant Physiol. 2001, 125, 1611-1619. [CrossRef]

31. Pérez-Munive, C.; Moreno Diaz de la Espina, S. Nuclear spectrin-like proteins are structural actin-binding proteins in plants. Biol. Cell 2011, 103, 145-157. [CrossRef]

32. Michaud, D.; Guillet, G.; Roger, P.A.; Charest, P.M. Identification of a 220-kDa membrane-associated plant cell protein immunologically related to human $\beta$-spectrin. FEBS Lett. 1991, 294, 77-80. [CrossRef]

33. De Ruijter, N.C.A.; Emons, A.M.C. Immunodetection of spectrin in plant cells. Cell Biol. Int. 1993, 17, 169-182. [CrossRef]

34. Khan, A.A.; Hanada, T.; Mohseni, M.; Jeong, J.J.; Zeng, L.; Gaetani, M.; Li, D.; Reed, B.C.; Speicher, D.W.; Chishti, A.H. Dematin and adducing provide a novel link between the spectrin cytoskeleton and human erythrocyte membrane by directly interacting with glucose transporter-1. J. Biol. Chem. 2008, 283, 14600-14609. [CrossRef] [PubMed]

35. Salomao, M.; Zhang, X.; Yang, Y.; Lee, S.; Hartwig, J.H.; Chasis, J.A.; Mohandas, N.; An, X. Protein 4.1R-dependent multiprotein complex: New insights into the structural organization of the red blood cell membrane. Proc. Natl. Acad. Sci. USA 2008, 105, 8026-8031. [CrossRef] [PubMed]

36. Machnicka, B.; Grochowalska, R.; Bogusławska, D.M.; Sikorski, A.F.; Lecomte, M.C. Spectrin-based skeleton as an actor in cell signaling. Cell Mol. Life Sci. 2012, 69, 191-201. [CrossRef] [PubMed]

37. Haslam, R.J.; Koide, H.B.; Hemmings, B.A. Pleckstrin domain homology. Nature 1993, 363, 309-310. [CrossRef] [PubMed]

38. Musacchio, A.; Gibson, T.; Rice, P.; Thompson, J.; Saraste, M. The PH domain: A common piece in the structural patchwork of signaling proteins. Trends Biochem. Sci. 1993, 18, 343-348. [CrossRef]

39. Macias, M.J.; Musacchio, A.; Ponstingl, H.; Nilges, M.; Saraste, M.; Oschkinat, H. Structure of the pleckstrin homology domain from beta-spectrin. Nature 1994, 369, 675-677. [CrossRef] [PubMed]

40. DeWald, D.B.; Torabinejad, J.; Jones, C.A.; Shope, J.C.; Cangelosi, A.R.; Thompson, J.E.; Prestwich, G.D.; Hama, H. Rapid accumulation of phosphatidylinositol 4,5-bisphosphate and inositol 1,4,5-trisphosphate correlates with calcium mobilization in salt-stressed Arabidopsis. Plant Physiol. 2001, 126, 759-769. [CrossRef]

41. Ulm, R.; Baumann, A.; Oravecz, A.; Máté, Z.; Ádám, É.; Oakeley, E.J.; Nagy, F. Genome-wide analysis of gene expression reveals function of the bZIP transcription factor HY5 in the UV-B response of Arabidopsis. Proc. Natl. Acad. Sci. USA 2004, 101, 1397-1402. [CrossRef]

42. Favory, J.J.; Stec, A.; Gruber, H.; Rizzini, L.; Oravecz, A.; Funk, M.; Seidlitz, H.K. Interaction of COP1 and UVR8 regulates UV-B-induced photomorphogenesis and stress acclimation in Arabidopsis. EMBO J. 2009, 28, 591-601. [CrossRef]

43. Jakoby, M.; Weisshaar, B.; Dröge-Laser, W.; Vicente-Carbajosa, J.; Tiedemann, J.; Kroj, T.; Parcy, F. bZIP transcription factors in Arabidopsis. Trends Plant Sci. 2002, 7, 106-111. [CrossRef] 
44. Murmu, J.; Bush, M.J.; DeLong, C.; Li, S.; Xu, M.; Khan, M.; Hepworth, S.R. Arabidopsis basic leucine-zipper transcription factors TGA9 and TGA10 interact with floral glutaredoxins ROXY1 and ROXY2 and are redundantly required for anther development. Plant Physiol. 2010, 154, 1492-1504. [CrossRef] [PubMed]

45. Shigeoka, S.; Maruta, T. Cellular redox regulation, signaling, and stress response in plants. Biosci. Biotechnol. Biochem. 2014, 78, 1457-1470. [CrossRef] [PubMed]

46. Noshi, M.; Mori, D.; Tanabe, N.; Maruta, T.; Shigeoka, S. Arabidopsis clade IV TGA transcription factors, TGA10 and TGA9, are involved in ROS-mediated responses to bacterial PAMP flg22. Plant Sci. 2016, 252, 12-21. [CrossRef] [PubMed]

47. Gunawardena, H.P.; Huang, Y.; Kenjale, R.; Wang, H.; Xie, L.; Chen, X. Unambiguous characterization of site-specific phosphorylation of leucine-rich repeat Fli-I-interacting protein 2 (LRRFIP2) in toll-like receptor 4 (TLR4)-mediated signaling. J. Biol. Chem. 2011, 286, 10897-10910. [CrossRef] [PubMed]

48. Ye, Y.; Ding, Y.; Jiang, Q.; Wang, F.; Sun, J.; Zhu, C. The role of receptor-like protein kinases (RLKs) in abiotic stress response in plants. Plant Cell Rep. 2017, 36, 235-242. [CrossRef]

49. Fu, S.F.; Chen, P.Y.; Nguyen, Q.T.T.; Huang, L.Y.; Zeng, G.R.; Huang, T.L.; Lin, C.Y.; Huang, H.J. Transcriptome profiling of genes and pathways associated with arsenic toxicity and tolerance in Arabidopsis. BMC Plant Biol. 2014, 14, 94. [CrossRef]

50. Chang, G.J.; Hwang, S.G.; Yong, C.P.; Hyeon, M.P.; Dong, S.K.; Duck, H.P.; Cheol, S.J. Molecular characterization of the cold- and heat-induced Arabidopsis PXL1 gene and its potential role in transduction pathways under temperature fluctuations. J. Plant Physiol. 2015, 176, 138-146. [CrossRef]

51. Ma, X.L.; Cui, W.N.; Zhao, Q.; Zhao, J.; Hou, X.N.; Li, D.Y.; Chen, Z.L.; Shen, Y.Z.; Huang, Z.J. Functional study of a salt-inducible TaSR gene in Triticum aestivum. Physiol. Plant. 2015, 156, 40-53. [CrossRef]

52. Wu, F.; Sheng, P.; Tan, J.; Chen, X.L.; Lu, G.W.; Ma, W.W.; Heng, Y.Q.; Lin, Q.B.; Zhu, S.S.; Wang, J.L.; et al. Plasma membrane receptor-like kinase leaf panicle 2 acts downstream of the drought and salt tolerance transcription factor to regulate drought sensitivity in rice. J. Exp. Bot. 2015, 66, 271-281. [CrossRef]

53. Caldwell, M.M. Solar UV irradiation and the growth and development of higher plants. Photophysiology 1971, 6, 131-177. [CrossRef]

54. Elshire, R.J.; Glaubitz, J.C.; Sun, Q.; Poland, J.A.; Kawamoto, K.; Buckler, E.S.; Mitchellet, S.E. A robust, simple genotyping-by-sequencing (GBS) approach for high diversity species. PLoS ONE 2011, 6, e19379. [CrossRef] [PubMed]

55. Langmead, B.; Salzberg, S.L. Fast gapped-read alignment with Bowtie 2. Nat. Methods 2012, 9, 357-359. [CrossRef] [PubMed]

56. Glaubitz, J.C.; Casstevens, T.M.; Lu, F.; Harriman, J.; Elshire, R.J.; Sun, Q.; Buckler, E.S. TASSEL-GBS: A high capacity genotyping by sequencing analysis pipeline. PLoS ONE 2014, 9, e90346. [CrossRef] [PubMed]

57. Livak, K.J.; Schmittgen, T.D. Analysis of relative gene expression data using real-time quantitative PCR and the $2^{-\Delta \Delta C}$ T method. Methods 2001, 25, 402-408. [CrossRef] [PubMed]

58. Grant, D.; Nelson, R.T.; Cannon, S.B.; Shoemaker, R.C. SoyBase, the USDA-ARS soybean genetics and genomics database. Nucleic Acids Res. 2010, 38, D843-D846. [CrossRef] [PubMed]

59. Wang, Y.; Tang, H.; DeBarry, J.D.; Tan, X.; Li, J.; Wang, X.; Lee, T.; Jin, H.; Marler, B.; Hui, G.; et al. MCScanX: A toolkit for detection and evolutionary analysis of gene synteny and collinearity. Nucleic. Acids Res. 2012, 40, e49. [CrossRef]

(C) 2019 by the authors. Licensee MDPI, Basel, Switzerland. This article is an open access article distributed under the terms and conditions of the Creative Commons Attribution (CC BY) license (http://creativecommons.org/licenses/by/4.0/). 